\title{
Evaluation on the analogy between the dynamic magnetosphere and a forced and/or self-organized critical system
}

\author{
A. T. Y. Lui \\ The Johns Hopkins University Applied Physics Laboratory, Laurel, Maryland 20723, USA
}

Received: 19 November 2001 - Accepted: 11 December 2001

\begin{abstract}
The dissipation power and size of auroral blobs are investigated in detail to examine the possible analogy between the dynamic magnetosphere and a forced and/or selforganized critical system. The distributions of these auroral parameters are sorted in terms of different levels of activity, namely substorms, pseudo-breakups, and quiet conditions. A power law (scale-free) component is seen in all these distributions. In addition, a peak distribution is found for substorm intervals and a hump for pseudo-breakup intervals. The peak distribution is present prominently during magnetic storms, i.e. when the magnetosphere is strongly driven by the solar wind. It is interpreted that the scale-free component is associated with the activity of the diffuse aurora, corresponding to disturbances at all permissible scales within the plasma sheet. Ionospheric feedback appears to be essential for the presence of two components in the distribution for auroral dissipation power. These results are consistent with the concept that the magnetosphere is in a forced and/or self-organized critical state, although they do not constitute conclusive evidence for the analogy.
\end{abstract}

\section{Introduction}

As an intermediate region between the solar wind and the Earth's ionosphere, the Earth's magnetosphere not only shields the Earth from the direct impact of the solar wind, but also couples tightly the ionosphere with the solar wind. In addition, the magnetosphere is a huge reservoir of energetic charged particles from both the solar wind and the ionosphere. A crude estimate based on the dimensions of the magnetosphere and the averaged number density gives the order of $10^{31}$ particles residing within the magnetosphere. Interestingly, in spite of the enormous number of degrees of freedom expected for the large number of particles present within the magnetosphere system, the global behaviour of the magnetosphere seems to depend on just a few parameters. In

Correspondence to: A. T. Y. Lui (Tony.Lui@jhuapl.edu) fact, reconstructing dynamics from time series data, such as the $A E$ index, leads to the result indicating the global magnetospheric dynamics to be low dimensional and predictable (e.g. Vassiliadis et al., 1990). This has led some to view the magnetosphere as a deterministic chaotic system.

The low dimension aspect of the magnetosphere is not unique to a deterministic chaotic system, however. It is pointed out by Chang $(1992,1998,1999)$ that a system near criticality is also low dimensional. In addition, he suggested that the power law spectrum of magnetic fluctuations and intermittent turbulent features may be signatures of the magnetosphere being an open, dissipative dynamical system at a critical state, dubbed as the forced and/or self-organized criticality (FSOC) state. The concept of self-organized criticality (SOC) was first introduced by Bak et al. (1987), who showed that slowly driven dynamical systems, with many degrees of freedom, naturally self-organize into a critical state, with avalanches of all sizes obeying power law statistics. The critical state is an attractor for the dynamics and the phenomenon is deterministic and robust with respect to noise and inhomogeneities. While SOC requires no tuning of its driver, in the version of Chang, a slight fine-tuning of the driver is needed to get the system near the critical point, hence, FSOC instead of SOC. This concept seems to fit well with some earlier work expressing the view of intermittent localized disturbances constituting the basic element of magnetospheric activity (e.g. Lui, 1991; Angelopoulos et al., 1992) and with the results from a number of analysis relating to the $A E$ index (e.g. Consolini, 1997a; Consolini et al., 1996).

In this paper, we shall examine the magnetosphere from one of its energy outputs, namely the auroral activity seen in the polar regions. Reported here is an extension of the previous work along this line of research (Lui et al., 2000). The distributions of auroral power and size of luminosity obtained from a satellite-borne imager, the UltraViolet Imager (UVI), on the Polar satellite will be examined in detail. Distributions specific to the strong external driving condition of the magnetosphere, namely during magnetic storms, will also be examined for comparison with other times. Finally, the 
robustness of the distributions in auroral power and size is tested with a large range of thresholding on the auroral luminosity in the construction of the distributions.

\section{Dissipation power and size of auroral blobs}

The energy output of the magnetosphere manifested by auroras in the polar region was examined with global images from Polar UVI. The analysis was carried out in several steps as done in the previous study (Lui et al., 2000). First, the raw images from Polar UVI were transformed into Cartesian coordinates of magnetic latitude and magnetic local time. The luminosity was then corrected for background by subtracting the luminosity outside the auroral region. The power and size of individual auroral disturbances, called hereafter as auroral blobs, in each image were determined by applying an intensity threshold. In this study, we extend the previous work by sorting into periods of substorms, pseudo-breakups, and quiet times. Pseudo-breakup periods are those in which transient and localized brightening of aurora was observed, but the activity did not develop into the characteristic global auroral pattern as in magnetospheric substorms. This sequence of analyses was performed for the entire set of auroral images in the entire month of January 1997.

Figure 1 shows the normalized occurrence histograms of auroral dissipation power from binning auroral blobs in the three activity categories, together with the overall histogram regardless of the activity level. There are two interesting features. First is the occurrence of a power law component in all histograms. In other words, the auroral blobs exhibit a scale-free component in all these categories. Furthermore, the power spectral index appears to have the same value regardless of the activity level. Second, for intervals other than quiet times, there is an additional component with a peak or hump in the histogram distribution, superposed on the scalefree component. This indicates that this second component does have a range for its characteristic scale. The median characteristic scale for substorm intervals is near $6 \times 10^{9}$ Watt. For pseudo-breakup intervals, there is a hump but not a prominent peak in the histogram. The hump occurs at a smaller value of the auroral dissipation power than that of the peak during substorms. This is understandable because pseudo-breakup events are expected to dissipate less power than that of substorms.

Figure 2 shows the normalized occurrence histograms of auroral size from binning the auroral blobs in the same manner as sorting the auroral dissipation power. It is noted that the upper cutoff on the size is due to the limited total viewing scope of UVI. Once again, the two components are present in all categories except for quiet times. The power spectral index for the scale-free component is similar in the four histograms shown. For the component with a peak during substorms, the median characteristics' scale appears to be near $2 \times 10^{6} \mathrm{~km}^{2}$.

\section{Strongly driven conditions}

Magnetic storms occur when the magnetosphere is driven strongly by the solar wind. One can examine the robustness of the features noted in auroral blobs under these extreme conditions. Figure 3 shows the auroral dissipation power (top row) and size (bottom row) during the main and recovery phases of magnetic storms. The period examined covers the 6-month interval from December 1996 to May 1997. The same two components are found as noted previously. The general features in these histograms are very similar to those shown before. A significant difference between these histograms and the ones shown in Figs. 1 and 2 is the location of the peak in the histograms. The characteristic scales associated with the peak in the histograms for storm main phase are significantly larger in both the auroral dissipation power and size than those in Figs. 1 and 2. This difference is noticeable also in the storm recovery phase, although it is not as significant. This result is expected since magnetic storms are large-scale events, involving more energy dissipation and spanning over a larger area.

\section{Effect of different thresholding}

Figure 4 shows the effects on the occurrence histograms of auroral dissipation power and area from a range of thresholds chosen corresponding to levels expected for being recognized as active aurora. These are the threshold levels explored in the earlier study (Lui et al., 2000). It is seen that the main features noted in Figs. 1 and 2 remain unchanged even though at the highest threshold the peaks in auroral power and size evolve into humps instead. The power spectral index for the power law component appears unchanged for auroral dissipation power, but increases gradually with higher thresholds for auroral size. If the threshold is taken to much higher levels, changes in these features can be noted.

Figure 5 displays the results from several high thresholds in comparison with the one used in Fig. 4. It is seen that the power spectral index for the scale-free component in the auroral dissipation power distribution remains unchanged. However, the peak in the distribution becomes less and less pronounced and shifts progressively to lower values as the threshold level is raised. This trend also occurs for the auroral size. On the other hand, the power law component in the auroral size distribution remains unchanged but has a significantly steeper slope than those at lower thresholds.

\section{Summary and discussion}

In this article, the auroral activity is used as a means to monitor the energy output of the magnetosphere and to examine the statistical characteristics of magnetospheric disturbances in relation to the concept of forced and/or self-organized criticality. We have extended the previous analysis of auroral blobs in several ways. First, we include statistics of power 

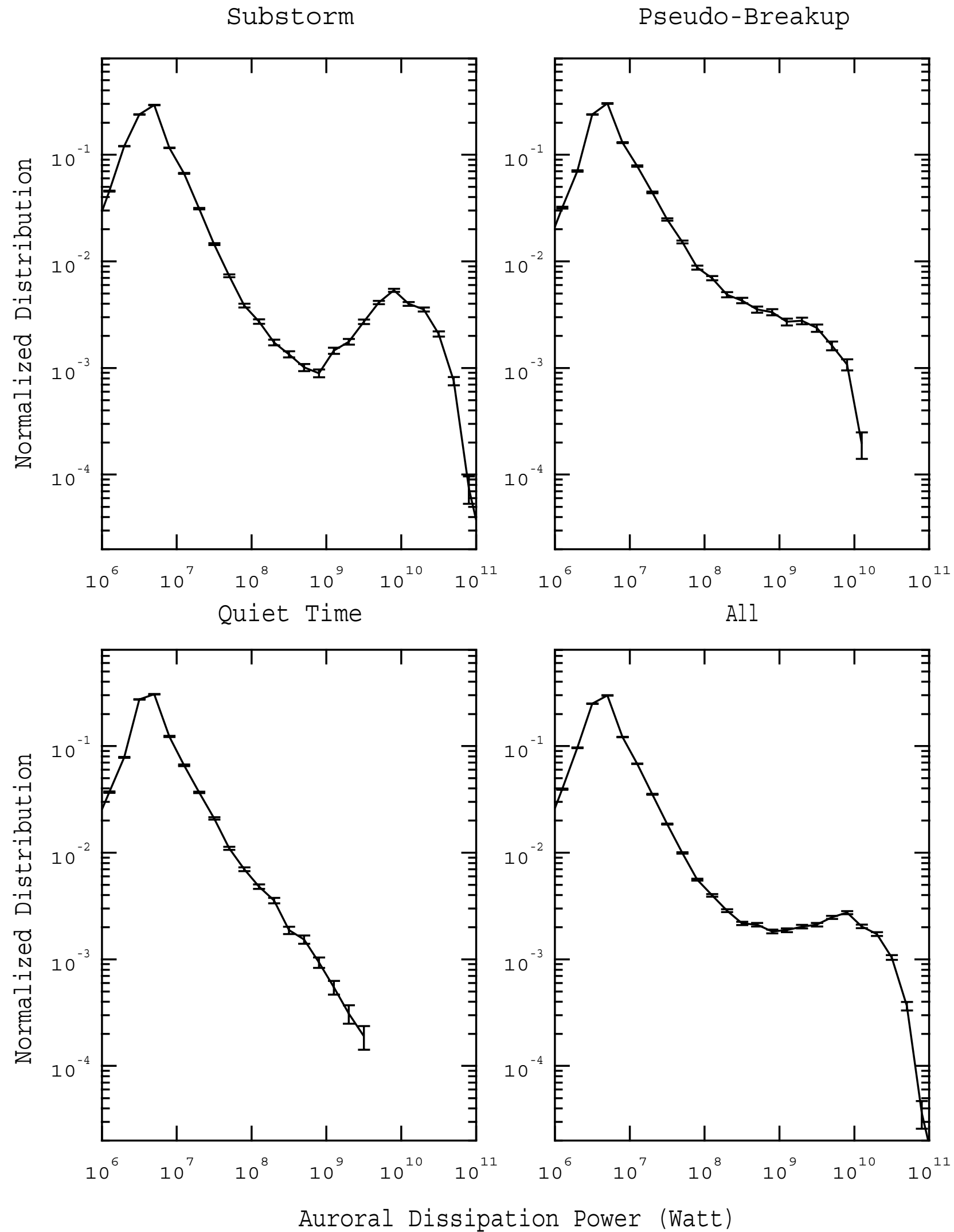

Fig. 1. Normalized occurrence frequency of dissipation power in auroral blobs for intervals of substorm activity, pseudo breakups, quiet times, and all conditions. 
Substorm Pseudo-Breakup
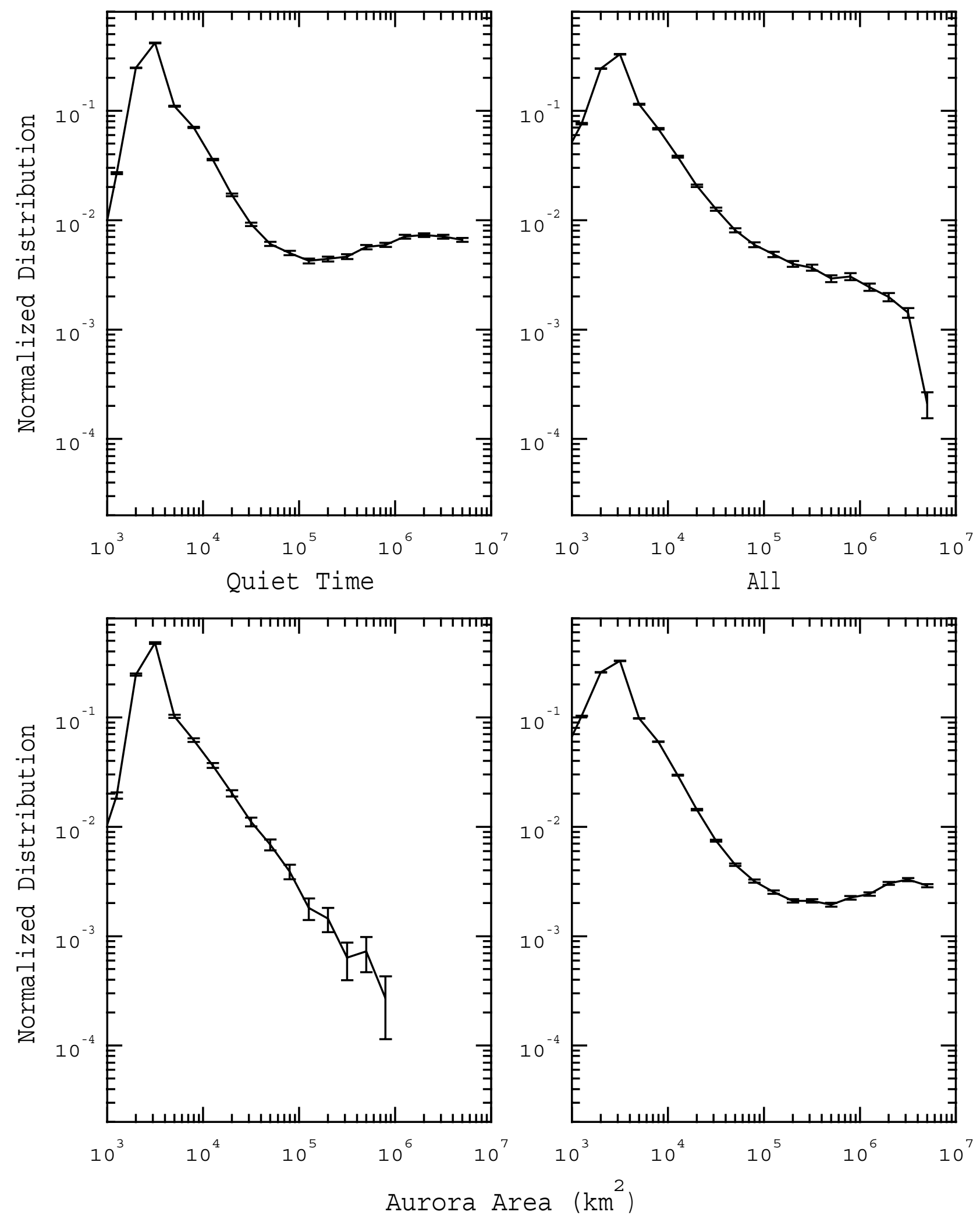

Fig. 2. Normalized occurrence frequency of size of auroral blobs for intervals of substorm activity, pseudo breakups, quiet times, and all conditions. 

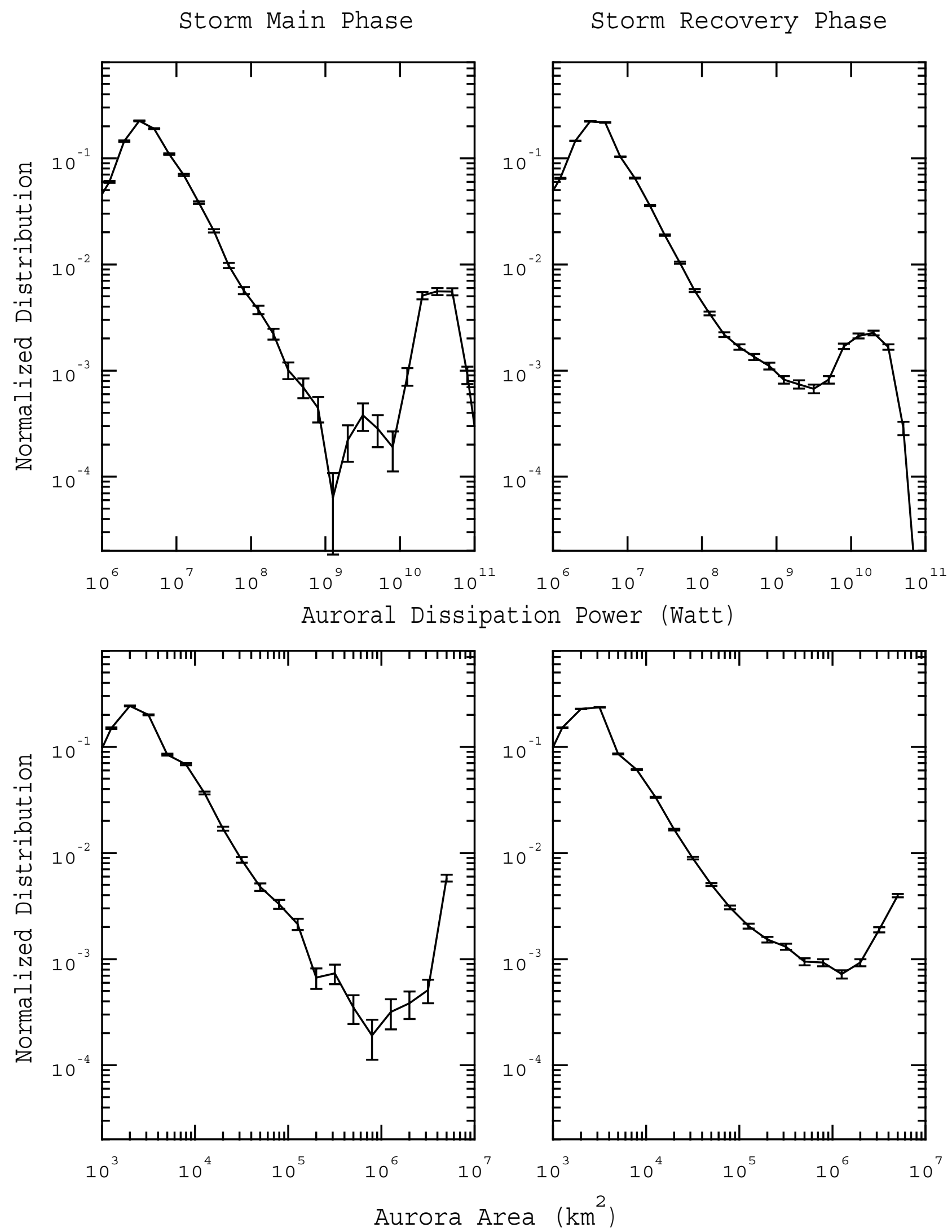

Fig. 3. Normalized occurrence frequency of dissipation power and size of auroral blobs during storm main phase and recovery phase. 
Substorm
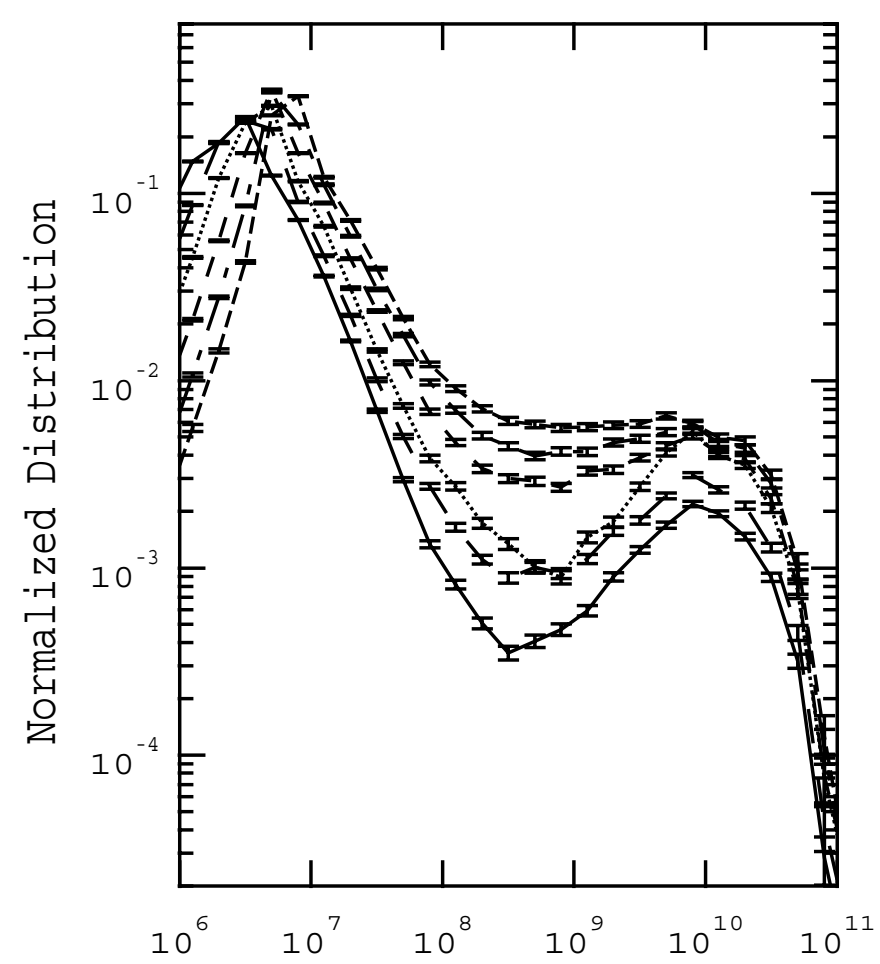

Quiet Time

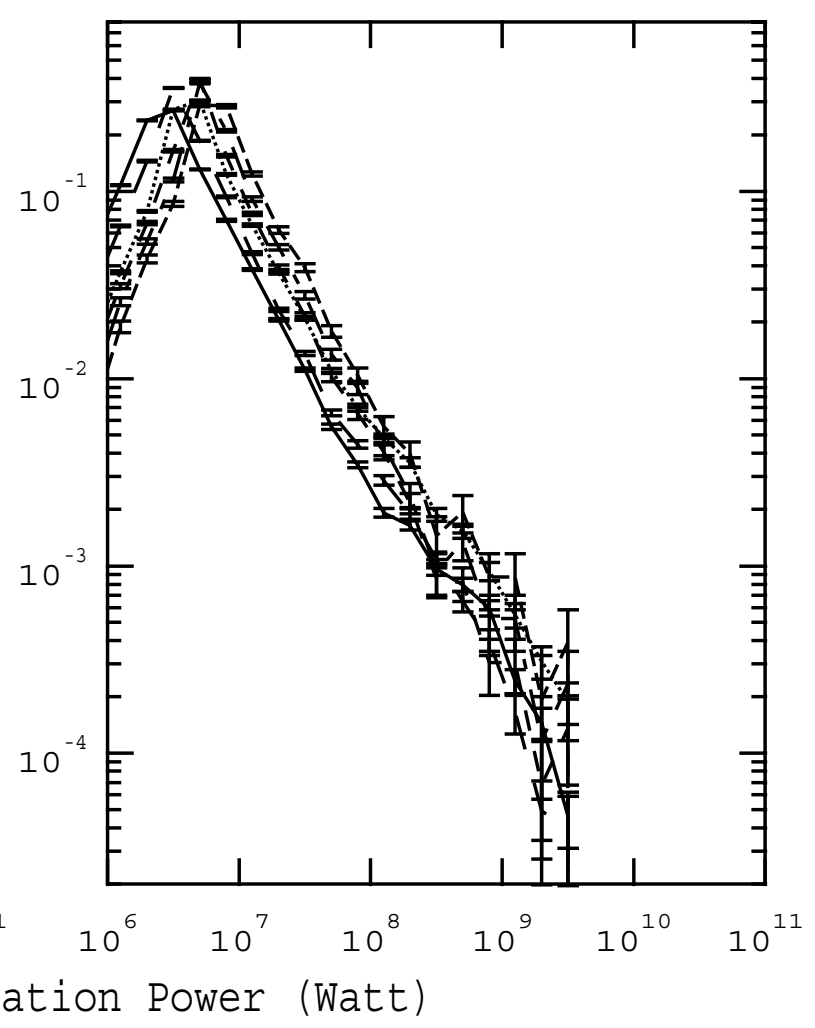

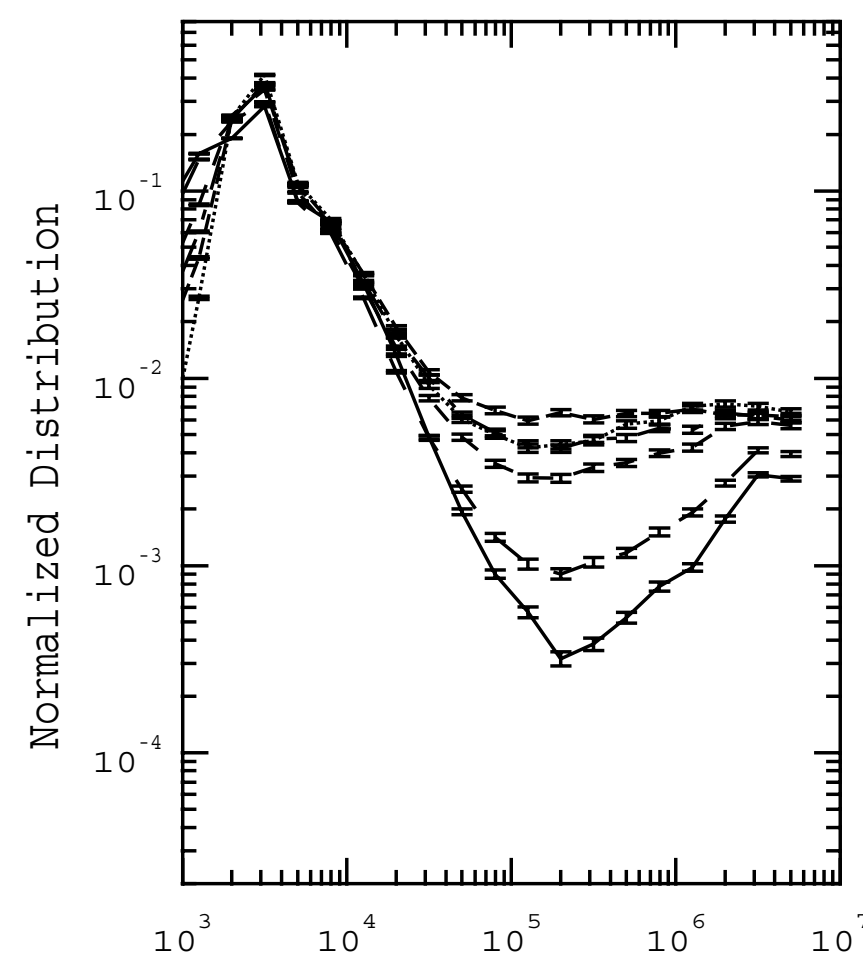

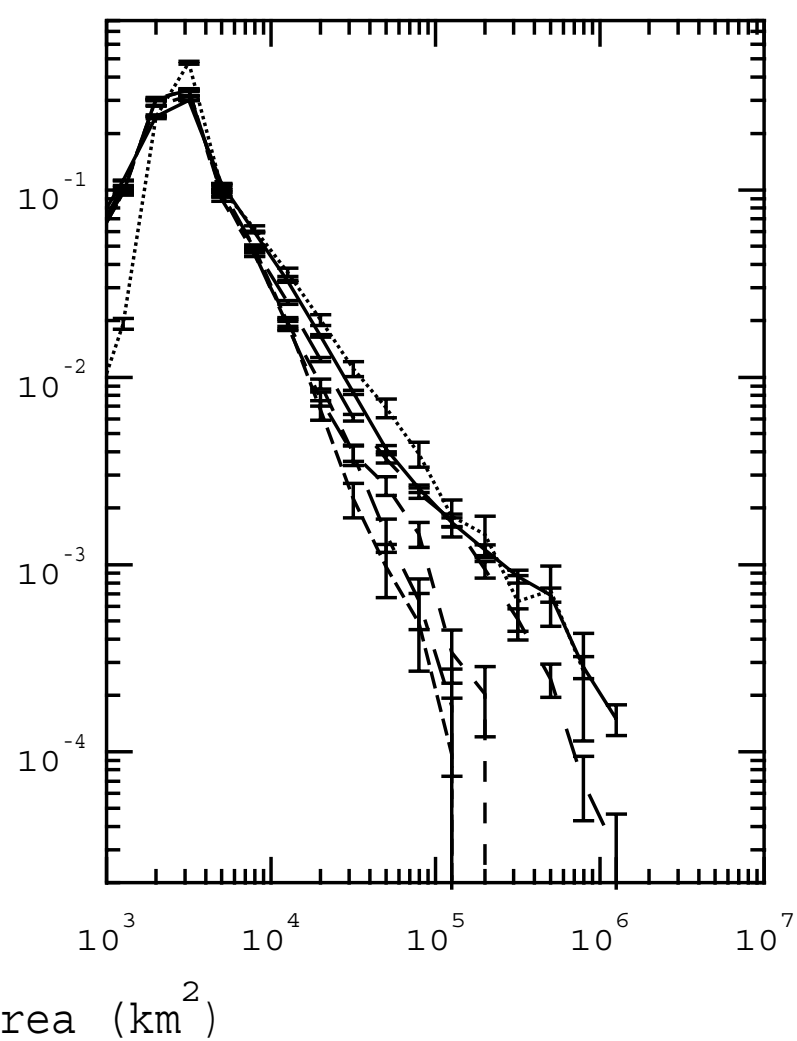

Fig. 4. Normalized occurrence frequency of dissipation power and size of auroral blobs for a nominal range of thresholds used in determining auroral blobs. The energy thresholds used are: $0.8,1.1,1.4,1.7,2.0$, and $2.3 \mathrm{erg} / \mathrm{cm}^{2} / \mathrm{s}$. 
Substorm

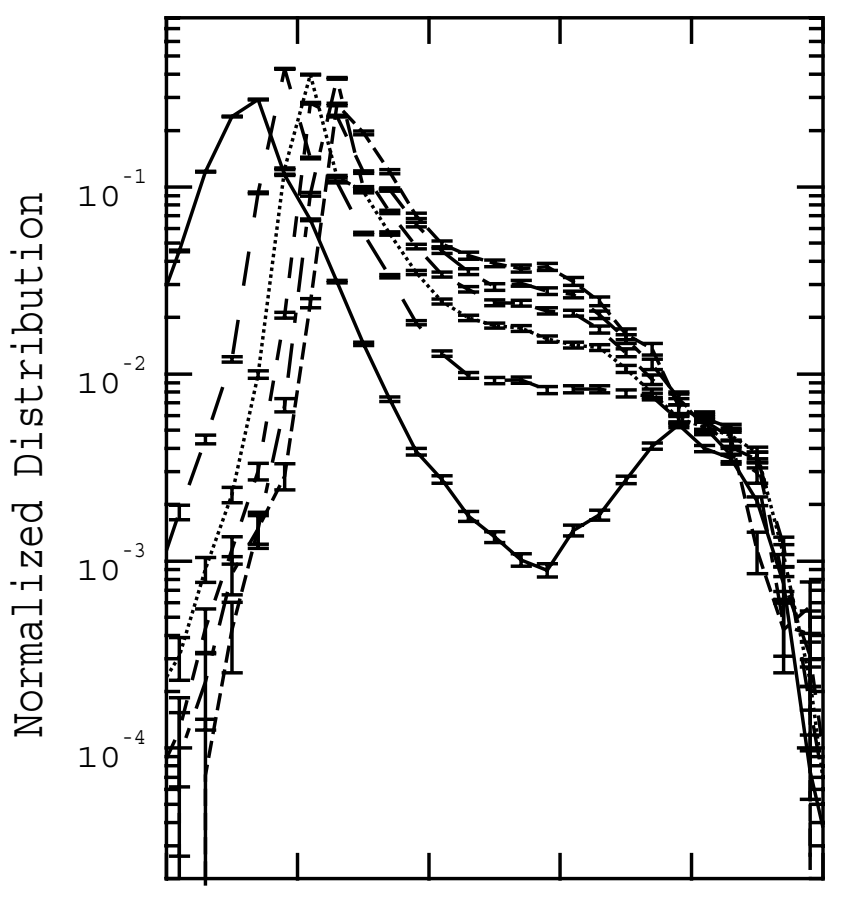

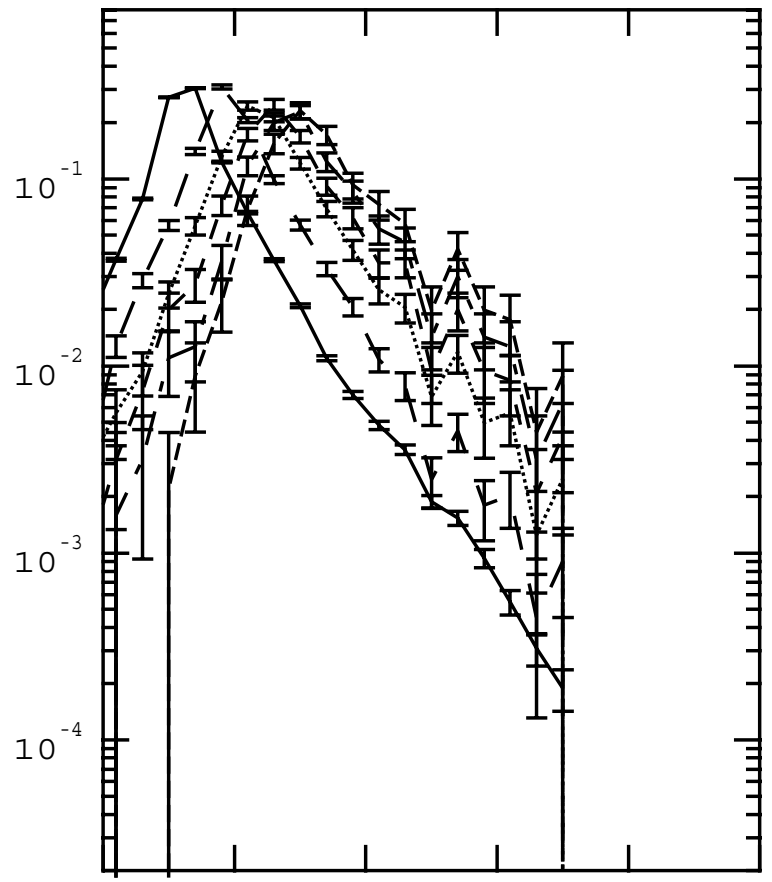

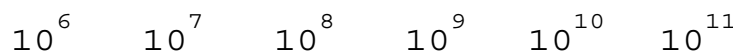

Auroral Dissipation Power (Watt)
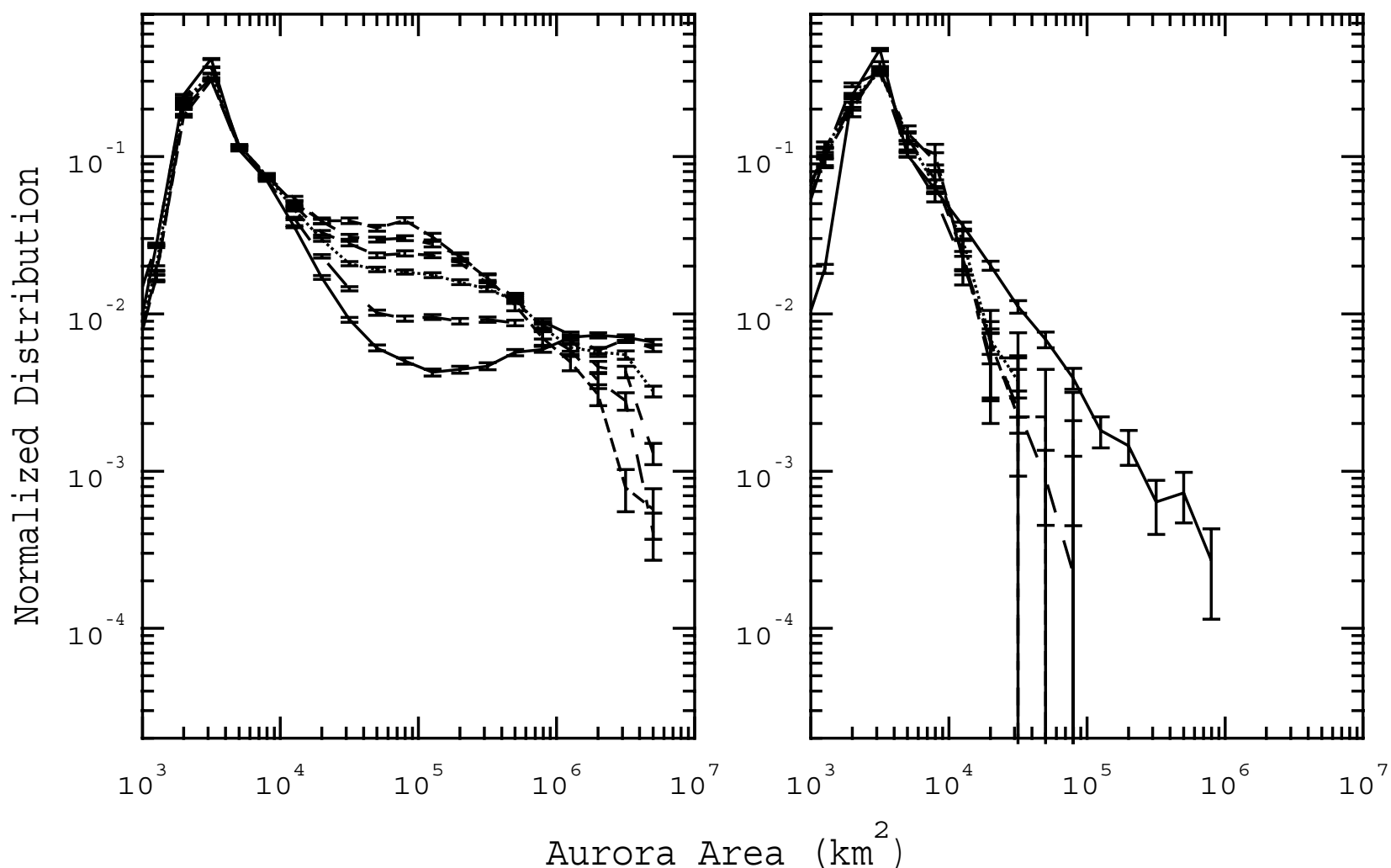

Fig. 5. Normalized occurrence frequency of dissipation power and size of auroral blobs for an extreme range of thresholds used in determining auroral blobs. The energy thresholds used are: 1.4 (for comparison with Fig. 4), 2.8, 4.1, 5.5, 6.8, and $8.2 \mathrm{erg} / \mathrm{cm}^{2} / \mathrm{s}$. 
dissipation and size of auroral blobs for pseudo-breakup intervals as well as for all samples. We also isolate these statistics for main and recovery phases of magnetic storms, i.e. conditions when the magnetosphere is being strongly driven by the solar wind. The robustness of features in these statistics is examined through a large range of thresholds in the determination of auroral blobs.

In the early study, the presence of a peak in the occurrence frequency of dissipation power and size was compared with the results from a simple sandpile model. The component in the histograms for substorms, which shows a peak, was interpreted as being constituted by disturbances with a longrange correlation, corresponding to system-wide avalanches in the numerical sandpile model. However, since a noticeable hump exists in histograms for pseudo-breakup intervals in which disturbances are localized and thus, are not "systemwide" disturbance, this previous interpretation may need to be modified. One possible interpretation is that both the pseudo-breakup and substorm events have a range of characteristic scales, where the scale associated with the former is significantly less than the latter. The scale-free component found in all situations probably corresponds to perpetual disturbances of all permissible scale sizes occurring within the plasma sheet. The auroral manifestation of the plasma sheet is the diffuse aurora. The fact that the component with a characteristic scale gradually disappears as the threshold for blob determination is raised to very high levels indicates that the long-range correlation in the plasma sheet for high levels of activity diminishes gradually with increasing threshold levels.

Watkins et al. (1999) have studied the robustness of collective behaviour in strongly driven avalanche models. They found that there is a lower cutoff of energy output for sandpiles under constant strong driving. This feature disappears when the strong driving is variable. From the statistics of storm intervals, no lower cutoff of power dissipation and size is seen. Therefore, the results found here for storms could be interpreted as consistent with the magnetosphere to be analogous to an avalanching system under variable strong driving.

Recently, Kozelov and Kozelova (2001) pointed out that the comparison of distributions for auroral dissipation power with that of avalanche energy may not be appropriate. Indeed, the work by Chapman et al. (1998) did not design the simulation to allow for the determination of the duration of each avalanche. Thus, separation of instantaneous power and total energy of each avalanche cannot be made in their model. On the other hand, Kozelov and Kozelova (2001) did perform a numerical sandpile model, allowing for the separation between instantaneous power and total energy of avalanche. From this simulation, they found that the distribution for avalanche power in the SOC model does not have a power law form. Furthermore, they found it necessary to include a parameter in each cell which mimics the ionospheric feedback to the magnetosphere. For situations when the ionospheric feedback is below a critical threshold for all cells, the distribution shows a power law over a limited range. For situations when the ionospheric feedback exceeds a critical threshold in at least one cell, the distribution shows two components resembling auroral dissipation power during substorms. Their interpretation is that the component which shows a range of characteristic scales indicates times when ionospheric feedback is important in the overall dynamics of the system.

In conclusion, the observed features in the analysis of auroral dissipation power and size are quite consistent with the view that the magnetosphere behaves like a forced and/or self-organized critical system, as suggested by a number of previous works (e.g. Chang, 1992, 1998, 1999; Consolini et al., 1996; Consolini, 1997b; Chapman et al., 1998; Kozelov and Kozelova, 2001). However, these findings cannot be considered as providing conclusive evidence for the magnetosphere acting as a system near a critical point, since there are systems other than FSOC which have similar characteristics as found here (Watkins et al., 2001). Furthermore, Freeman et al. (2000) have noted that the external energy input parameters in the solar wind have a similar shape of distributions as the auroral electrojet indices. The solar wind parameters they examined are $v B_{s}$ and $\epsilon$, where $v$ is the solar wind speed, $B_{S}$ is the southward component of the interplanetary magnetic field, and $\epsilon$ is a parameter introduced by Perrault and Akasofu (1978) which combines the solar wind velocity with the east-west and north-south components of the interplanetary magnetic field. They argued that one possibility is that the scale-free component may have originated from the external source. They attributed the SOC features in the auroral indices to be a result of the solar wind being a SOC system. Given these uncertainties, it is clear that more analysis on other parameters of the magnetosphere is needed in the future to establish firmly the validity in drawing the analogy between the magnetosphere and a FSOC system.

Acknowledgement. This work was supported by NASA Grants NAG5-10475, NAG5-7797 and the Atmospheric Sciences Division of NSF Grant ATM-0000219 to the Johns Hopkins University Applied Physics Laboratory.

\section{References}

Angelopoulos, V., Baumjohann, W., Kennel, C. F., Coroniti, F. V., Kivelson, M. G., Pellat, R., Luhr, H., and Paschmann, G.: Bursty bulk flow in the inner central plasma sheet, J. Geophys. Res., 97, 4027-4039, 1992.

Bak, P., Tang, C., and Wiesenfeld, K.: Self-organized criticality: An explanation of 1/f noise, Phys. Rev. Lett., 59, 381-384, 1987.

Chang, T.: Low dimensional behaviour and symmetry breaking of stochastic systems near criticality - can these effects be observed in space and in the laboratory?, EEE Trans. Plasma Sci., 20, 691694, 1992.

Chang, T.: Sporadic localized reconnection and multiscale intermittent turbulence in the magnetotail, AGU Monograph No. 104, Geospace Mass and Energy Flow, (Eds) Horwitz, J. L., Gallagher, D. L., and Peterson, W. K., p. 193, (American Geophysical Union, Washington, D. C.), 1998. 
Chang, T.: Self-organized criticality, multi-fractal spectra, sporadic localized reconnections and intermittent turbulence in the magnetotail, Physics of Plasmas, 6, 4137-4145, 1999.

Chapman, S. C., Watkins, N. W., Dendy, R. O., Helander, P., and Rowlands, G.: A simple avalanche model as an analogue for magnetospheric activity, Geophys. Res. Lett., 25, 2397-2400, 1998.

Consolini, G.; Intermittency and turbulence in magnetospheric dynamics, Proceedings of the International School of Physics "Enrico Fermi”, (Eds) Mallamace, F. and Stanley, H. E., IOS Press, Amsterdam, 657, 1997a.

Consolini, G.: Sandpile cellular automata and magnetospheric dynamics, in: Proceedings of Cosmic Phyiscs in the year 2000, (Eds) Aiello et al., 58, SIF, Bologna, Italy, 1997b.

Consolini, G., Marcucci, M. F., and Candidi, M.: Multifractal structure of auroral electrojet index data, Phys. Rev. Lett., 76 (21), 4082-4085, 1996.

Freeman, M. P., Watkins, N. W., and Riley, D. J.: Evidence for a solar wind origin of the power law burst lifetime distribution of the $A E$ indices, Geophys. Res. Lett., 27, 1087-1090, 2000.

Kozelov, B. V. and Kozelova, T. V.: Sandpile model analogy of the magnetosphere-ionosphere substorm activity, Proc. Interball Meeting, Warsaw, Poland, 2001.

Lui, A. T. Y.: Plasma transport in the Earth's magnetotail, Modeling Magnetospheric Plasma Processes, ed. by G. R. Wilson, 41-53, 1991.

Lui, A. T. Y., Chapman, S. C., Liou, K., Newell, P. T., Meng, C.-I., Brittnacher, M., and Parks, G. K.: Is the dynamic magnetosphere an avalanching system?, Geophys. Res. Lett., 27, 911-914, 2000.

Perreault, P. and Akasofu, S.-I.: A study of geomagnetic storms, Geophys. J. R. Astron. Soc., 54, 547-573, 1978.

Vassiliadis, D. V., Sharma, A. S., Eastman, T. E., and Papadopoulos, K.: Low-dimensional chaos in magnetospheric activity from $A E$ time series, Geophys. Res. Lett., 17, 1841-1844, 1990.

Watkins, N. W., Chapman, S. C., Dendy, R. O., and Rowlands, G.: Robustness of collective behaviour in strongly driven avalanche models: Magnetospheric implications, Geophys. Res. Lett., 26, 2617-2620, 1999.

Watkins, N. W., Freeman, M. P., Chapman, S. C., and Dendy, R. O.: Testing the SOC hypothesis for the magnetosphere, J. Atmos. and Solar Terr. Phys., 63, 1435-1445, 2001. 